\title{
Effect of Pretreatment Platelet Parameters on Survival in Limited Disease Small Cell Lung Cancer
}

\author{
Abdullah Sakin ${ }^{1 *}$, Nurgul Yasar², Serdar Arici², Cumhur Demir ${ }^{2}$, Caglayan \\ Geredeli $^{2}$, Ferdi Aksaray ${ }^{3}$, Selver Isik ${ }^{4}$, Sener Cihan ${ }^{2}$
}

\begin{abstract}
Background: The aim of this study was to investigate the effect of platelet parameters before concurrent chemoradiotherapy (CCRT) on survival of patients with limited disease small cell lung cancer (LD-SCLC). Methods: This study consisted of patients who received CCRT due to LD-SCLC in the oncology clinic between 1997-2017. Examined platelet parameters included total platelet count (TPC), mean platelet volume, platelet distribution width, and platelet-lymphocyte ratio. The cut-off value for TPC was determined as 306x10\%/U (sensitivity: $62 \%$, specificity: $75.5 \%$ ), where patients below or equal to this level was classified as Group I, and those above as Group II. Results:The study included 90 patients whose mean age was 59 years (range: $42-83$ ) and male ratio was $80.0 \%(\mathrm{n}=72)$. Near three-fourths of patients (74.4\%) were at clinical stage III. Among stage I-II patients, mOS was found as 126 months for Group I whereas it had not been reached in Group II $(\mathrm{p}=0.158)$. Stage III patients showed significantly lower mOS for Group 1 (16 [range: 14.1-17.8] months) compared to that in Group 2 (19.0 [range: 15.6-62.8] months; $\mathrm{p}=0.002$ ). In multivariate analysis, Eastern Cooperative Oncology Group performance score $(\mathrm{p}=0.003)$, clinical stage $(\mathrm{p}<0.001)$, prophylactic cranial irradiation $(\mathrm{p}=0.004)$, and TPC $(\mathrm{p}=0.031)$ was determined as the most significant factors affecting survival. Conclusion: Our study suggests association of high baseline levels of TPC to improved survival in patients scheduled to undergo CCRT for LD-SCLC. Considering easiness and universal availability of TPC measurement, potential utilization of this biomarker may be promising to predict survival, albeit requiring validation by further well-designated prospective studies.
\end{abstract}

Keywords: Small cell lung carcinoma- limited disease- chemoradiotherapy- total platelet count- survival

Asian Pac J Cancer Prev, 20 (6), 1879-1885

\section{Introduction}

The most common cause of cancer-related deaths worldwide is the lung cancer. Approximately 1.8 million patients were diagnosed with lung cancer and near 1.6 million lung cancer-related deaths occurred across the globe in 2012 (Siegel et al., 2018).

Small cell lung cancer (SCLC) accounts for about $15 \%$ of all lung cancers. It is clinically different from non-small cell lung cancer (NSCLC) because of its rapid progression and early development of metastases (Gaspar et al., 2005; Silvestri et al., 2013). Only 5\% of all SCLC patients are diagnosed at the limited disease (LD) stage of the disease; the remaining patients get the diagnosis at the extensive disease (ED) stage. Highly greater probability of ED at the time of diagnosis can be attributed to the high degree of aggressive biology, rapid tumor progression, absence of symptoms in the early stage, and lack of well-established screening programs. Chemoradiotherapy (CRT) remains the standard treatment modality in LD-SCLC (Chen et al., 2010; Varlotto et al., 2011). On the other hand, potential prognostic factors are still unclear in this patient subset.

Platelets are responsible for initiating hemostatic mechanisms that abolish vascular endothelial injury (Smyth et al., 2009). Several recent studies have reported that platelets were associated with development and progression of malignancy (Riedl et al., 2014; Franco et al., 2015). Activated platelets were suggested to promote cancer cell growth, angiogenesis, and invasion (Buergy et al., 2012). Furthermore, association of some platelets indices were reported to prognosis in various cancers, including NSCLC, breast cancer, colorectal cancer, gastric cancer, pancreatic cancer, and laryngeal cancer. Several of these parameters, including total platelet count (TPC), mean platelet volume (MPV), platelet distribution width (PDW), and platelet-lymphocyte ratio (PLR) can be easily tested (Song et al., 2017; Zhang et al., 2017a; Zhang et al., 2017b). Nevertheless, the findings of studies

${ }^{1}$ Department of Medical Oncology, Yuzuncu Yil University Medical School, Van, ${ }^{2}$ Department of Medical Oncology, ${ }^{3}$ Department of Radiation Oncology, University of Health Sciences, Okmeydani Training and Research Hospital, Istanbul, ${ }^{4}$ Department of Medical Oncology, University of Healt Sciences, Erzurum Bolge Training and Research Hospital, Erzurum, Turkey. *For Correspondence: drsakin@hotmail.com 
reporting the association of platelet counts and other platelet indices to the presence and progression of cancer are still controversial and suggest the need for clarification (Monreal et al., 1998; Liu et al., 2017; Pedrazzani et al., 2017; Zhang et al., 2019).

This study aimed to investigate the effect of selected pretreatment platelet indices on survival of patients with LD-SCLC.

\section{Materials and Methods}

\section{Patients}

This retrospective study included patients who received concurrent chemoradiotherapy (CCRT) due to LD-SCLC in the oncology clinic between 1997-2017. Staging of patients were performed according to findings of pre-treatment fluorodeoxyglucose positron emission tomography (PET-CT), computed tomography (CT), and cranial magnetic resonance imaging (MRI). Tumor size was calculated by measuring the largest tumor diameter in CT. Exclusion criteria were malignancy other than SCLC, or those having ED-SCLC, autoimmune disease, history of aspirin or any immunosuppressive drug use, being under the age of 18 , or whose data were not accessible. In our hospital, SCLC staging is performed based on PET-CT and cranial MRI findings, and pre-treatment clinical, pathological, and laboratory data are recorded. Ensuring a 10-12 hours of fasting, samples for blood tests are routinely taken from antecubital vein by establishing mild venous stasis at upper arm in patients who apply to oncology clinic before treatment. Blood samples for biochemical parameters are taken into the anticoagulant-free gel tubes, and those for complete blood counts into ethylenediamine tetraacetate-containing tubes. Complete blood count parameters are examined in hemogram autoanalyser (Mindray, China). Biochemical parameters are tested in autoanalyser (Beckman Coulter, USA) using colorimetric method. Based on Eastern Cooperative Oncology Group performance score (ECOG PS) and comorbidities, patients are treated with concomitant CRT of two cycles with platinum [cisplatin (60-75 mg/m2) or carboplatin (AUC 5)] + etoposide (100 $\mathrm{mg} / \mathrm{m} 2$ for 1-3 days) at 28-day intervals, followed with two to four cycles of the same chemotherapeutic regimen at 21-day intervals. Radiotherapy is administered as a total of $60 \mathrm{~Gy}$, consisting of 30 fractions as 2 Gy per day.

Data collection: Data on the age, gender, smoking status, ECOG PS, history of superior vena cava syndrome (SVCS), clinical tumor stage, chemotherapy regimen, cycle count, history of prophylactic cranial irradiation (PCI), presence of recurrence/metastasis, and survival status were obtained from medical records. In addition, initial laboratory values immediately after diagnosis were collected: creatinine, aspartate aminotransferase (AST), alanine aminotransferase (ALT), alkaline phosphatase (ALP), gamma glutamyl transferase (GGT), lactate dehydrogenase (LDH), sodium, potassium, calcium, albumin, white blood cell (WBC) count, red blood cell (RBC) count, hemoglobin $(\mathrm{Hb})$, hematocrit (Hct), mean corpuscular volume (MCV), total platelet count (TPC), total neutrophil count (TNC), total lymphocyte count (TLC), and total monocyte count (TMC). Neutrophil-lymphocyte ratio (NLR) was obtained by dividing the TNC by TLC. PLR was obtained by dividing TPC by TLC. Monocyte-lymphocyte ratio (MLR) was obtained by dividing the TMC by TLC. Patients were further stratified by their age as $<65$ years and $\geq 65$ years. Patients' ECOG PS were grouped as either $0-2$ or 3-4. Receiver operating characteristics (ROC) curves were formed for overall survival (OS) using TPC, PDW, and PLR data at diagnosis. The areas under the curve (AUCs) were detected as 0.736 (95\% CI 0.629-0.842, $\mathrm{p}<0.001)$, 0.580 (95\% Cl 0459-0702, $\mathrm{p}=0.197)$, and $0.405(95 \% \mathrm{Cl}$ $0.283-0,526, p=0.126)$, respectively. The cut-off value for TPC was determined as $306 \times 10^{9} / \mathrm{U}$ with a sensitivity of $62 \%$ and a specificity of $75.5 \%$ (Figure 1). Patients having a TLC of $\leq 306 \times 10^{9} / \mathrm{U}$ were categorized as Group I and those having a TLC of $>306 \times 10^{9} / \mathrm{U}$ were as Group II. OS was calculated as time from the diagnosis to the date of last follow-up or death. The study was performed in accordance with the declaration of Helsinki and was reviewed and approved by the Ethics Committee of the University of Health Sciences, Okmeydani Training and Research Hospital (26.2.18).

Statistical analysis: Statistical Package for the Social Sciences 22.0 for Windows software (Armonk NY, IBM Corp, 2013) was used for the statistical analysis. Descriptive statistics were presented as the mean, standard deviation, minimum, and maximum values for numerical variables. The comparison of the rates between the groups was performed by chi-square analysis. Numerical variable between two independent groups were analyzed with student t-test in case of normal distribution and with Mann Whitney U test if else. Monte Carlo simulation was applied if conditions could not be met. Survival analyses were performed with Kaplan-Meier Analysis. Determinant factors were examined with cox regression. Backward stepwise model was used with parameters having a p-value below 0.100 . An overall $5 \%$ alpha error level was used to infer statistical significance.

\section{Results}

The study included 90 patients whose mean age was 59 years (range: $42-83$ ), and male cases constituted $80.0 \%$ $(n=72)$. Twenty patients $(22.2 \%)$ were $\geq 65$ years old. Most of patients (95.6\%) had history of smoking. Thirteen patients $(14.4 \%)$ had ECOG PS of $\geq 2$. SVCS was present at time of diagnosis in six patients $(6.7 \%)$. A quarter of patients $(25.6 \%)$ were at clinical stage I or II. Patients received mostly $(91.1 \%)$ cisplatin + etoposide protocol, with a mean number of 4.9 cycles overall. PCI was performed in 50 patients $(55.6 \%)$. During the follow-up, $71(78.9 \%)$ patients had relapse/metastasis and $53(58.9 \%)$ patients had died. There were 54 patients $(60.0 \%)$ in Group I and 36 patients (40.0\%) in Group II. These study groups were found as similar in terms of age, gender, smoking status, ECOG PS, tumor localization, presence of SVCS, clinical stage, chemotherapy regimen, received cycle count, and relapse/metastasis rates (Table 1).

Group I had significantly higher levels of mean baseline Hb, Hct, and MPV ( $\mathrm{p}=0.004, \mathrm{p}=0.005$, and 
Table 1. Demographic and Clinical Characteristics of the Study Population

\begin{tabular}{|c|c|c|c|c|c|c|c|c|}
\hline & & \multicolumn{2}{|c|}{ All patient } & \multicolumn{2}{|c|}{ Group 1} & \multicolumn{2}{|c|}{ Group 2} & \multirow[b]{2}{*}{$\mathrm{p}$} \\
\hline & & $\mathrm{n}$ & $\%$ & $\mathrm{n}$ & $\%$ & $\mathrm{n}$ & $\%$ & \\
\hline \multirow[t]{2}{*}{ Gender } & Female & 18 & 20 & 8 & 14.8 & 10 & 27.8 & 0.132 \\
\hline & Male & 72 & 80 & 46 & 85.2 & 26 & 72.2 & \\
\hline \multirow[t]{2}{*}{ Age group } & $<65$ years & 70 & 77.8 & 41 & 75.9 & 29 & 80.6 & 0.605 \\
\hline & $\geq 65$ years & 20 & 22.2 & 13 & 24.1 & 7 & 19.4 & \\
\hline \multirow[t]{2}{*}{ Smoking status } & No & 4 & 4.4 & 1 & 1.9 & 3 & 8.3 & 0.144 \\
\hline & Yes & 86 & 95.6 & 53 & 98.1 & 33 & 91.7 & \\
\hline \multirow[t]{2}{*}{ ECOG PS } & $0-1-2$ & 77 & 85.6 & 44 & 81.5 & 33 & 91.7 & 0.178 \\
\hline & $3-4$ & 13 & 14.4 & 10 & 18.5 & 3 & 8.3 & \\
\hline \multirow[t]{2}{*}{ SVCS } & No & 84 & 93.3 & 51 & 94.4 & 33 & 91.7 & 0.605 \\
\hline & Yes & 6 & 6.7 & 3 & 5.6 & 3 & 8.3 & \\
\hline \multirow[t]{2}{*}{ Clinical stage } & $\mathrm{I}+\mathrm{II}$ & 23 & 25.6 & 11 & 20.4 & 12 & 33.3 & 0.167 \\
\hline & III & 67 & 74.4 & 43 & 79.6 & 24 & 66.7 & \\
\hline \multirow[t]{2}{*}{ Chemotherapy regimen } & Cisplatin + etoposide & 82 & 91.1 & 49 & 90.7 & 33 & 91.7 & 0.879 \\
\hline & Carboplatin + etoposide & 8 & 8.9 & 5 & 9.3 & 3 & 8.3 & \\
\hline \multirow[t]{2}{*}{ PCI } & No & 40 & 44.4 & 28 & 51.9 & 12 & 33.3 & 0.083 \\
\hline & Yes & 50 & 55.6 & 26 & 48.1 & 24 & 66.7 & \\
\hline \multirow[t]{2}{*}{ Relapse/metastasis } & No & 19 & 21.1 & 9 & 16.7 & 10 & 27.8 & 0.206 \\
\hline & Yes & 71 & 78.9 & 45 & 83.3 & 26 & 72.2 & \\
\hline \multirow[t]{2}{*}{ Final status } & Alive & 37 & 41.1 & 14 & 25.9 & 23 & 63.9 & $<0.001$ \\
\hline & Exitus & 53 & 58.9 & 40 & 74.1 & 13 & 36.1 & \\
\hline Age (years) & Mean (Min-Max) & \multicolumn{2}{|c|}{$59.0(42-83)$} & \multicolumn{2}{|c|}{$60.0(42-77)$} & \multicolumn{2}{|c|}{$58.0(46-83)$} & 0.166 \\
\hline Smoking (pack-years) & Mean \pm SD (Min-Max) & \multicolumn{2}{|c|}{$57.8 \pm 34.7(0-180)$} & \multicolumn{2}{|c|}{$57.6 \pm 30.8(0-180)$} & \multicolumn{2}{|c|}{$58.0 \pm 40.3(0-160)$} & 0.67 \\
\hline Tumor size (cm) & Mean \pm SD (Min-Max) & \multicolumn{2}{|c|}{$5.8 \pm 2.6(2.0-15.0)$} & \multicolumn{2}{|c|}{$5.4 \pm 2.2(2.0-10.5)$} & \multicolumn{2}{|c|}{$6.5 \pm 3.1(2.0-15.0)$} & 0.131 \\
\hline $\begin{array}{l}\text { The number of cycles } \\
\text { of chemotherapy }\end{array}$ & Mean \pm SD (Min-Max) & \multicolumn{2}{|c|}{$4.9 \pm 1.1(2-6)$} & \multicolumn{2}{|c|}{$4.8 \pm 1.2(2-6)$} & \multicolumn{2}{|c|}{$5.1 \pm 1.0(3-6)$} & 0.268 \\
\hline
\end{tabular}

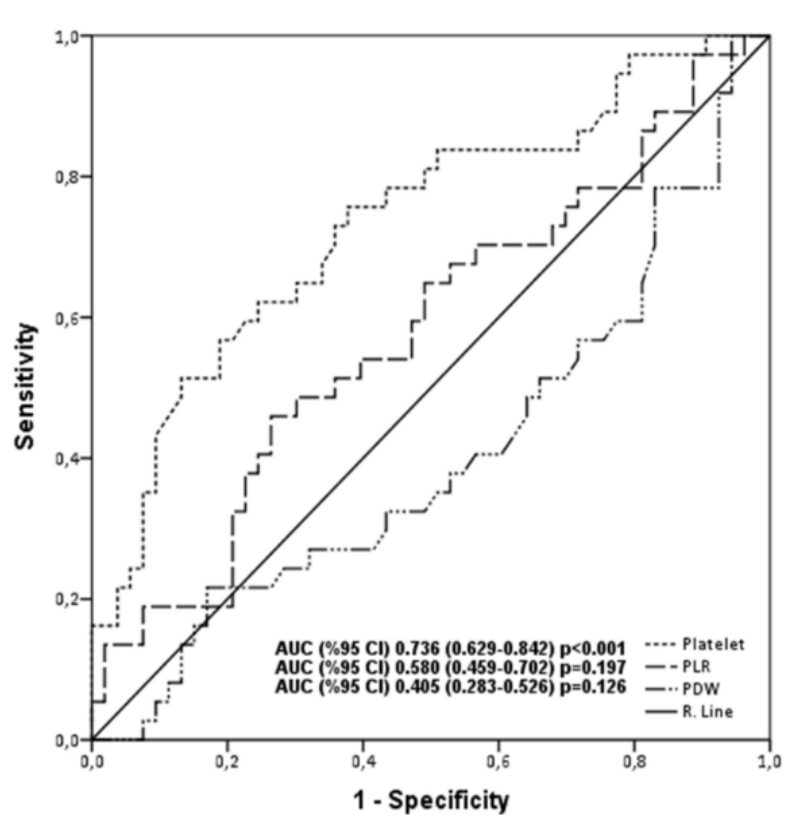

Figure 1. ROC Curve Analyses for OS; platelets are represented by the line with $\mathrm{AUC}=0.736(95 \% \mathrm{CI}$, $0.629-0.842 ; \mathrm{p}<0.001)$ with a sensitivity of $62.2 \%$ and $\mathrm{a}$ specificity of $75.5 \%(306 \times 103)$. AUC, the area under the curve, PDW, platelet distribution width, PLR, plateletto-lymphocyte ratio $\mathrm{p}=0.040$; respectively) and significantly lower levels of mean baseline WBC, TNC, and PLR ( $\mathrm{p}=0.011, \mathrm{p}=0.011$, and $p<0.001$; respectively) compared to those in Group II. All other laboratory values did not significantly differ between these study groups (Table 2).

Median overall survival (mOS) was significantly higher in patients with clinical stage I or II compared to that in patients with clinical stage III (126 [range: 29.2-281.5] vs. 19 [range: 16.3-31.6] months, respectively; log rank $\mathrm{p}<0.001)$. mOS in Group I (17 [range: 13.3-20.6] months) was significantly lower than that in Group II (70 [range: 21.3-118.6] months; log rank $\mathrm{p}<0.001)$. Among stage I-II patients, mOS was found as 126 months for Group I whereas it had not been reached in Group II (log rank $\mathrm{p}=0.158)$. Stage III patients showed significantly lower mOS for Group I (16 [range: 14.1-17.8] months) compared to that in Group II (19.0 [range: 15.6-62.8] months; log rank $\mathrm{p}=0.002$ ), (Figure 2).

Univariate analysis showed the age, ECOG PS, clinical stage, PCI, number of chemotherapy cycles, TPC, PDW, and PLR as the significant factors that affected survival $(\mathrm{p}=0.006, \mathrm{p}<0.001, \mathrm{p}<0.001, \mathrm{p}<0.001, \mathrm{p}=0.010, \mathrm{p}=0.002$, $\mathrm{p}=0.031$, and $\mathrm{p}=0.028$; respectively), (Table 3 ). In further multivariate analysis, Eastern Cooperative Oncology Group performance score $(\mathrm{p}=0.003)$, clinical stage 
Table 2. Baseline Laboratory Parameters of the Study Population

\begin{tabular}{|c|c|c|c|c|c|c|c|}
\hline & & All patient & & Group 1 & & Group 2 & \\
\hline & $\mathrm{n}$ & Mean \pm SD (Min-Max) & $\mathrm{n}$ & Mean \pm SD (Min-Max) & $\mathrm{n}$ & Mean \pm SD (Min-Max) & $\mathrm{p}$ \\
\hline Creatinine (mg/dL) & 84 & $0.8 \pm 0.2(0.3-1.5)$ & 49 & $0.8 \pm 0.2(0.5-1.5)$ & 35 & $0.8 \pm 0.2(0.3-1.3)$ & 0.114 \\
\hline $\operatorname{AST}(\mathrm{U} / \mathrm{L})$ & 79 & $22.4 \pm 12.6(9-79)$ & 47 & $24.1 \pm 14.3(10-79)$ & 32 & $19.8 \pm 9.0(9-60)$ & 0.24 \\
\hline $\operatorname{ALT}(\mathrm{U} / \mathrm{L})$ & 79 & $22.1 \pm 15.3(7-104)$ & 47 & $21.4 \pm 13.0(7-64)$ & 32 & $23.3 \pm 18.4(7-104)$ & 0.869 \\
\hline GGT (U/L) & 58 & $52.7 \pm 77.6(8-467)$ & 33 & $61.3 \pm 99.3(8-467)$ & 25 & $41.4 \pm 30.3(10-153)$ & 0.081 \\
\hline $\mathrm{LDH}(\mathrm{U} / \mathrm{L})$ & 57 & $259.3 \pm 119.4(120-671)$ & 31 & $259.2 \pm 101.9(120-560)$ & 26 & $259.5 \pm 139.5(120-671)$ & 0.718 \\
\hline $\operatorname{ALP}(\mathrm{U} / \mathrm{L})$ & 57 & $114.1 \pm 88.7(15-546)$ & 33 & $119.3 \pm 97.8(35-546)$ & 24 & $107.1 \pm 75.9(15-340)$ & 0.821 \\
\hline Sodium (mmol/L) & 43 & $139.3 \pm 3.3(130-146)$ & 25 & $139.0 \pm 3.7(130-145)$ & 18 & $139.7 \pm 2.6(134-146)$ & 0.804 \\
\hline Potassium (mmol/L) & 41 & $4.5 \pm 0.4(3.8-5.5)$ & 23 & $4.5 \pm 0.5(3.8-5.5)$ & 18 & $4.4 \pm 0.4(3.9-5.2)$ & 0.252 \\
\hline Calcium (mg/dL) & 40 & $9.5 \pm 0.4(8.5-10.7)$ & 25 & $9.6 \pm 0.5(8.5-10.7)$ & 15 & $9.5 \pm 0.2(9.0-9.9)$ & 0.474 \\
\hline WBC $\left(10^{9} / \mathrm{U}\right)$ & 90 & $8.9 \pm 3.5(2.5-25.5)$ & 54 & $8.3 \pm 3.2(2.5-21.1)$ & 36 & $9.9 \pm 3.9(4.4-25.9)$ & 0.011 \\
\hline $\operatorname{Rbc}\left(10^{9} / \mathrm{U}\right)$ & 90 & $4.5 \pm 0.6(3.0-6.4)$ & 54 & $4.6 \pm 0.7(3.0-6.4)$ & 36 & $4.4 \pm 0.4(3.4-5.2)$ & 0.076 \\
\hline $\mathrm{Hb}(\mathrm{g} / \mathrm{dL})$ & 90 & $12.9 \pm 1.7(9.3-17.3)$ & 54 & $13.3 \pm 1.8(9.3-17.3)$ & 36 & $12.3 \pm 1.5(9.7-15.2)$ & 0.004 \\
\hline Hct (\%) & 90 & $39.2 \pm 5.0(27.6-49.5)$ & 54 & $40.3 \pm 5.2(27.6-49.5)$ & 36 & $37.5 \pm 4.2(30.1-46.0)$ & 0.005 \\
\hline MCV (fL) & 90 & $86.7 \pm 6.8(64.1-102.2)$ & 54 & $87.6 \pm 6.5(67.1-102.2)$ & 36 & $85.3 \pm 7.2(64.1-98.6)$ & 0.12 \\
\hline TPC $\left(10^{9} / \mathrm{U}\right)$ & 90 & $302.9 \pm 128.3(38.8-798.0)$ & 54 & $222.4 \pm 56.8(38.8-305.0)$ & 36 & $423.6 \pm 109.7$ (307-798) & $<0.001$ \\
\hline PDW (\%) & 90 & $14.8 \pm 7.9(8.2-64.4)$ & 54 & $16.0 \pm 9.8(8.2-64.4)$ & 36 & $13.0 \pm 3.0(8.2-17.4)$ & 0.124 \\
\hline MPV (fL) & 90 & $8.9 \pm 1.5(5.3-13.1)$ & 54 & $9.2 \pm 1.6(5.8-13.1)$ & 36 & $8.4 \pm 1.4(5.3-10.4)$ & 0.04 \\
\hline RDW (\%) & 81 & $14.5 \pm 1.7(12.0-19.4)$ & 48 & $14.5 \pm 1.9(12.1-19.4)$ & 33 & $14.4 \pm 1.5(12.0-19.2)$ & 0.893 \\
\hline $\mathrm{TNC}\left(10^{9} / \mathrm{U}\right)$ & 90 & $6.1 \pm 3.2(1.6-23.6)$ & 54 & $5.5 \pm 2.8(1.6-18.5)$ & 36 & $6.8 \pm 3.6(2.4-23.6)$ & 0.011 \\
\hline $\operatorname{TLC}\left(10^{9} / \mathrm{U}\right)$ & 90 & $1.9 \pm 0.8(0.4-3.4)$ & 54 & $1.9 \pm 0.8(0.4-3.4)$ & 36 & $2.0 \pm 0.7(0.5-3.4)$ & 0.424 \\
\hline TMC $\left(10^{9} / \mathrm{U}\right)$ & 90 & $0.7 \pm 0.3(0.1-2.0)$ & 54 & $0.6 \pm 0.3(0.1-1.4)$ & 36 & $0.7 \pm 0.3(0.4-2.0)$ & 0.168 \\
\hline NLR & 90 & $3.5 \pm 2.8(1.1-20.1)$ & 54 & $3.3 \pm 2.5(1.1-16.8)$ & 36 & $3.9 \pm 3.3(1.1-20.1)$ & 0.236 \\
\hline PLR & 90 & $180.4 \pm 120.8(19.4-761.1)$ & 54 & $138.5 \pm 77.6(19.4-455.6)$ & 36 & $243.2 \pm 145.6(100.6-761.1)$ & $<0.001$ \\
\hline MLR & 90 & $0.4 \pm 0.3(0.1-2.0)$ & 54 & $0.4 \pm 0.2(0.1-1.3)$ & 36 & $0.4 \pm 0.3(0.2-2.0)$ & 0.595 \\
\hline
\end{tabular}

ALT, alanine aminotransferase; AST, aspartate aminotransferase; ALP, alkaline phosphatase; Hb, hemoglobin; Hct, hematocrit; GGT, gamma glutamyl transferase; LDH, lactate dehydrogenase; MCV, mean corpuscular volume; MLR, monocyte-to-lymphocyte ratio; MPV, mean platelet volume; NLR; neutrophil-to-lymphocyte ratio; PDW, platelet distribution width; PLR, platelet-to-lymphocyte ratio; RBC, red blood cell; RDW, red cell distribution width; TLC, total lymphocyte count; TMC, total monocyte count; TNC, total neutrophil count; TPC, total platelet count; WBC, white blood cell.

$(\mathrm{p}<0.001)$, prophylactic cranial irradiation $(\mathrm{p}=0.004)$, and TPC $(p=0.031)$ was determined as the most significant factors affecting survival, (Table 4).

\section{Discussion}

In this study, we investigated the effect of pre-treatment TPC, MPV, PDW, and PLR on survival of LD-SCLC patients managed with CRT. Interestingly, a higher TPC $\left(>306 \times 10^{3}\right)$ was associated with improved survival in clinical stages I to III.

SCLC is known to have a more aggressive behavior than does NCSLC. In recent years, mean life expectancy has been improved owing to novel targeted therapy and immunotherapy modalities (Gadgeel et al., 2018; Uemura and Hida, 2018). However, survival in SCLC has not been much changed despite improvements in treatment options in the last 15 years (Gaspar et al., 2012). A recent study reported 2 months of improved survival with add-on atezolizumab to chemotherapy in ED-SCLC (Horn et al., 2018). All these indicate that the need for the development of simple and feasible prognostic factors in SCLC is extremely important.

Inflammatory biomarkers reflect the responses of the host against malignant cells. Lately, there is a growing interest of the host's inflammatory response against the tumor. Several studies have proposed NLR, PLR, and TPC as indicators of inflammatory conditions, and these indicators were described as both prognostic and predictive biomarkers in some cancers (Monreal et al., 1998; Forget et al., 2013; Lee et al., 2013; Pedrazzani et al., 2017; Song et al., 2017; Zhang et al., 2017a).

Currently, there are many articles discussing the effect of thrombocytosis on survival in NSCLC (Pedersen and Milman, 1996; Aoe et al., 2004; Tomita et al., 2009; Hong et al., 2015; Xie et al., 2015; Hong et al., 2018). However, there is scarce data on the prognostic importance of pre-treatment of TPC, MPV, PDW, and PLR in SCLC. Previous studies have reported mainly unfavorable or no impact of high platelet counts on survival of patients with NSCLC (Pedersen and Milman, 1996; Aoe et al., 2004; Tomita et al., 2009; Cakar et al., 2011).

Xie et al., (2015) in their study of 383 Chinese LD-SCLC patients reported low $\mathrm{Hb}$, high PLR and NLR to be associated with poor prognosis; and determined important prognostic variables as PLR, age, smoking cessation, radiotherapy, chemotherapy, surgery, and PCI. Zhang et al., (2019) compared patients with high PLR $(>152.1)$ to those with lower levels in their study regarding 286 Chinese LD-SCLC patients, and found 
DOI:10.31557/APJCP.2019.20.6.1879

Platelet Parameters in Limited Disease Small Cell Lung Cancer

Table 3. Univariate Analysis for OS

\begin{tabular}{|c|c|c|c|c|}
\hline Variable & & HR & $95 \% \mathrm{CI}$ & $\mathrm{p}$ \\
\hline Gender & male vs. female & 1.512 & $0.711-3.215$ & 0.283 \\
\hline Age & years & 1.045 & $1.013-1.078$ & 0.006 \\
\hline Smoking status & no vs. yes & 1.824 & $0.438-7.599$ & 0.409 \\
\hline ECOG PS & $2-4$ vs. $0-1$ & 4.393 & $2.258-8.545$ & $<0.001$ \\
\hline Clinical stage & stage 3 vs. $1-2$ & 6.167 & $2.579-14.746$ & $<0.001$ \\
\hline PCI & yes vs. no & 0.327 & $0.188-0.570$ & $<0.001$ \\
\hline The number of cycles of chemotherapy & $\leq 4$ vs. $>4$ & 0.483 & $0.277-0.842$ & 0.01 \\
\hline Smoking & pack-years & 0.999 & $0.992-1.006$ & 0.826 \\
\hline Tumor size & $\mathrm{cm}$ & 0.92 & $0.820-1.032$ & 0.156 \\
\hline Creatinine & $\mathrm{mg} / \mathrm{dL}$ & 1.661 & $0.415-6.647$ & 0.473 \\
\hline AST & $\mathrm{U} / \mathrm{L}$ & 1.008 & $0.987-1.029$ & 0.463 \\
\hline ALT & $\mathrm{U} / \mathrm{L}$ & 0.999 & $0.977-1.022$ & 0.954 \\
\hline GGT & $\mathrm{U} / \mathrm{L}$ & 1.003 & $1.000-1.007$ & 0.081 \\
\hline LDH & $\mathrm{U} / \mathrm{L}$ & 1 & 0.997-1.002 & 0.81 \\
\hline ALP & $\mathrm{U} / \mathrm{L}$ & 0.999 & 0.994-1.004 & 0.689 \\
\hline Sodium & $\mathrm{mmol} / \mathrm{L}$ & 0.963 & $0.855-1.085$ & 0.534 \\
\hline Potassium & $\mathrm{mmol} / \mathrm{L}$ & 2.602 & $0.998-6.785$ & 0.055 \\
\hline Calcium & $\mathrm{mg} / \mathrm{dL}$ & 0.556 & $0.181-1.710$ & 0.306 \\
\hline WBC & $10^{9} / \mathrm{U}$ & 0.971 & $0.896-1.053$ & 0.478 \\
\hline $\mathrm{RBC}$ & $10^{9} / \mathrm{U}$ & 0.936 & $0.582-1.505$ & 0.785 \\
\hline $\mathrm{Hb}$ & $\mathrm{g} / \mathrm{dL}$ & 1.057 & $0.905-1.236$ & 0.483 \\
\hline Hct & $\%$ & 1.017 & $0.962-1.074$ & 0.556 \\
\hline $\mathrm{MCV}$ & $\mathrm{fL}$ & 1.025 & $0.983-1.070$ & 0.24 \\
\hline TPC & $10^{9} / \mathrm{U}$ & 0.996 & 0.994-0.999 & 0.002 \\
\hline PDW & $\%$ & 1.033 & $1.003-1.065$ & 0.031 \\
\hline MPV & $\mathrm{fL}$ & 1.092 & $0.917-1.300$ & 0.325 \\
\hline RDW & $\%$ & 1.102 & $0.921-1.318$ & 0.29 \\
\hline TNC & $10^{9} / \mathrm{U}$ & 0.971 & $0.891-1.058$ & 0.5 \\
\hline TLC & $10^{9} / \mathrm{U}$ & 1.031 & $0.700-1.517$ & 0.878 \\
\hline TMN & $10^{9} / \mathrm{U}$ & 1.054 & $0.401-2.770$ & 0.915 \\
\hline NLR & & 0.979 & $0.893-1.075$ & 0.662 \\
\hline PLR & & 0.997 & 0.994-1.000 & 0.028 \\
\hline MLR & & 1.186 & $0.410-3.432$ & 0.753 \\
\hline
\end{tabular}

ALT, alanine aminotransferase; AST, aspartate aminotransferase; ALP, alkaline phosphatase; ECOG PS, Eastern Cooperative Oncology Group; Hb, hemoglobin; Hct, hematocrit; GGT, gamma glutamyl transferase; LDH, lactate dehydrogenase; MCV, mean corpuscular volume; MLR, monocyteto-lymphocyte ratio; MPV, mean platelet volume; NLR; neutrophil-to-lymphocyte ratio; PCI, prophylactic cranial irradiation; PDW, platelet distribution width; PLR, platelet-to-lymphocyte ratio; RBC, red blood cell; RDW, red cell distribution width; SVCS, superior vena cava syndrome; TLC, total lymphocyte count; TMC, total monocyte count; TNC, total neutrophil count; TPC, total platelet count; WBC, white blood cell.

mOS as 19.5 vs. 27.4 months, respectively; and concluded pre-treatment PLR as an independent variable for OS.

Bernhardt et al., (2018) performed a study with 350 German LD-SCLC patients managed with CRT, where

Table 4. Multivariate Analysis for OS

\begin{tabular}{lcccc}
\hline Variable & & HR & $95 \%$ CI & $\mathrm{p}$ \\
\hline ECOG PS & 2-4 vs. 0-1 & 2.849 & $1.441-5.633$ & 0.003 \\
Clinical stage & 3 vs. 1-2 & 5.542 & $2.277-13.489$ & $<0.001$ \\
PCI & Yes vs. No & 0.447 & $0.248-0.805$ & 0.004 \\
TPC & $10^{9} / \mathrm{U}$ & 0.997 & $0.995-0.999$ & 0.031 \\
\hline
\end{tabular}

ECOG PS, Eastern Cooperative Oncology Group; PCI, prophylactic cranial irradiation; TPC, total platelet count. they reported no effect of NLR, age, $\mathrm{Hb}$, and TPC on survival. The authors stated that multivariate analysis showed PCI, LDH, and CRP as independent factors affecting survival. Suzuki et al., (2019) in their study of 122 LD-SCLC patients who received CRT and followed up between 2002 and 2015, detected that lymphopenia was an independent poor prognostic factor.

Liu et al., (2017) determined NLR but not PLR as an independent prognostic factor in their study of 139 Chinese patients (55 LD-SCLC, 83 ED-SCLC). Hong et al., (2015) reported PLR, NLR, Hb, and MCV as significant prognostic factors in multivariate analysis in their study of 919 Chinese patients consisting of 552 LD-SCLC and 352 ED-SCLC cases, where the former 

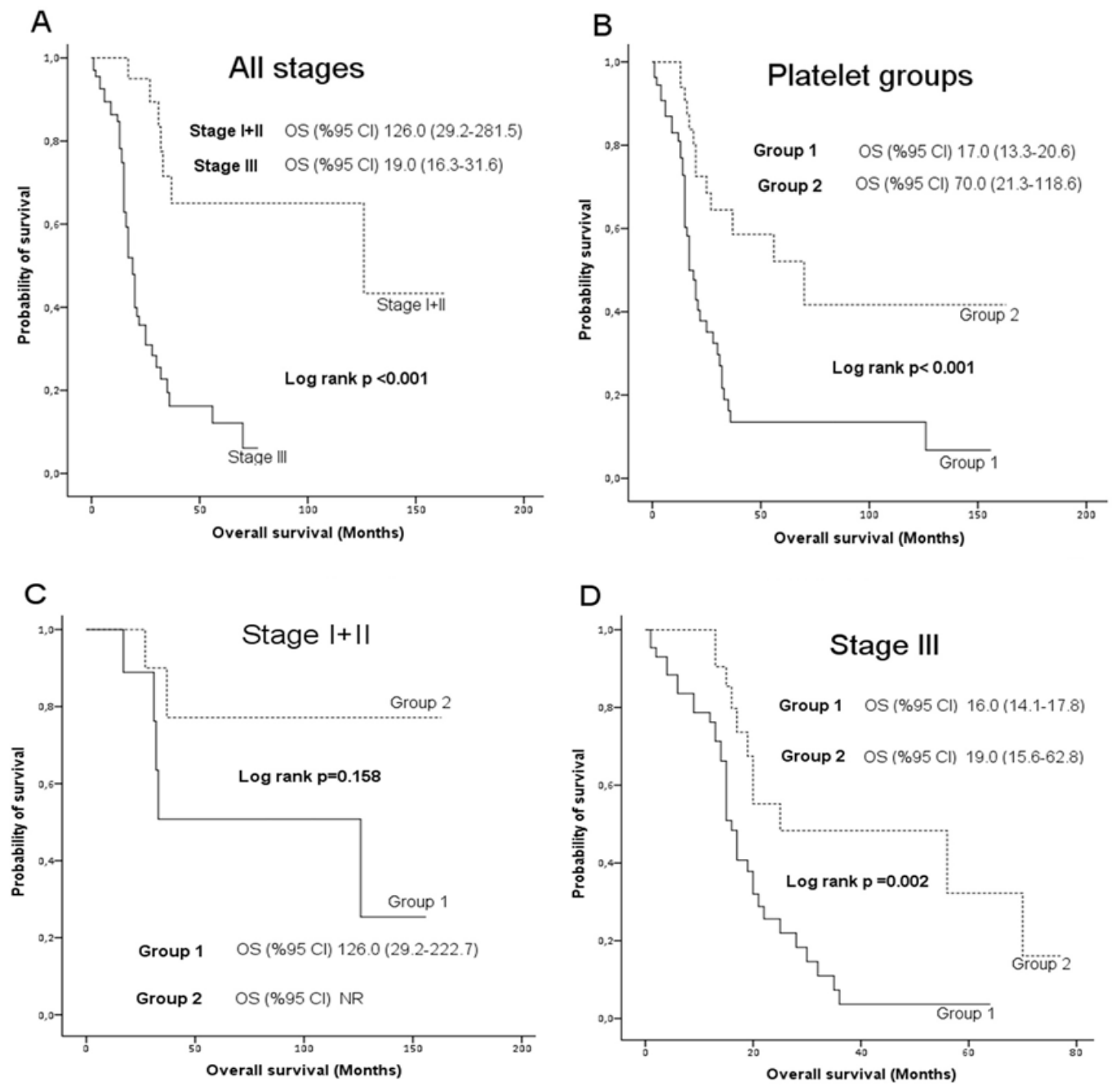

Figure 2. Overall Survival by (A) Clinical Stages, (B) Platelet Groups; (C) Platelet Groups in Stage I+II, and (D) Platelet Groups in Stage III.

group had a mOS of 11.8 months (Hong et al., 2015). Another study by Hong et al., (2018) among the 590 patients with LD-SCLC showed LDH, number of cycles of chemotherapy, and recurrence but not TPC as the independent factors affecting OS. Similarly, Deng et al. performed a study with 320 Chinese patients (122 LD-SCLC and 198 ED-SCLC), where they reported pre-treatment increased LDH and NLR but not PLR as an independent poor prognostic factor (Deng et al., 2017).

In our study, ECOG PS, clinical stage, PCI, and TPC were found to be independent factors affecting survival. All other laboratory parameters including NLR, PLR, MLR, TLC, MPV, and PDW were not associated with OS. The majority of studies about SCLC were performed on Chinese patients, among which treated with CRT had a mOS 11.2 to 27.4 months (Hong et al., 2015; Xie et al., 2015; Deng et al., 2017; Liu et al., 2017; Hong et al., 2018; Zhang et al., 2019). In our study, mOS was found as 25 months in all patients, including 126 months in stage I or II and 19 months in stage III. We did not measure the impact of ethnicity on our findings. For instance, German patients who received treatment with CRT had a mOS of 48 months (Kasmann et al., 2017).

Our study has several limitations. Although our study was performed only with LD-SCLC patients that received concomitant CRT with platinum + etoposide, we only included Turkish patients in a single-center setting with retrospective fashion. We cannot fully explain the mechanism of the effect of TPC on patients' prognosis in LD-SCLC. We also do not know the degree of impact of ethnicity on our conclusions. We believe that our results warrant confirmation by studies involving more comprehensive studies with larger sample size.

In conclusion, our study suggests association of elevated TPC before treatment to prognosis in patients who received concomitant CRT with platinum + etoposide due LD-SCLC. Considering easiness and universal availability of TPC measurement, potential utilization of this biomarker may be promising to predict survival, albeit requiring validation by further well-designated prospective studies.

\section{Funding Source}

None.

\section{Conflict-of-intereststatement}

All authors declare no conflicts-of-interest related to this article. 


\section{References}

Aoe K, Hiraki A, Ueoka H, et al (2004). Thrombocytosis as a useful prognostic indicator in patients with lung cancer. Respiration, 71, 170-3.

Bernhardt D, Aufderstrasse S, Konig L, et al (2018). Impact of inflammatory markers on survival in patients with limited disease small-cell lung cancer undergoing chemoradiotherapy. Cancer Manag Res, 10, 6563-9.

Buergy D, Wenz F, Groden C, et al (2012). Tumor-platelet interaction in solid tumors. Int $J$ Cancer, 130, 2747-60.

Cakar B, Karaoglanoglu M, Sayici Y, et al (2011). The prognostic value of thrombocytosis in newly diagnosed lung cancer patients: a retrospective analysis. $J$ BUON, 16, 677-81.

Chen J, Jiang R, Garces YI, et al (2010). Prognostic factors for limited-stage small cell lung cancer: a study of 284 patients. Lung Cancer, 67, 221-6.

Deng M, Ma X, Liang X, et al (2017). Are pretreatment neutrophil-lymphocyte ratio and platelet-lymphocyte ratio useful in predicting the outcomes of patients with small-cell lung cancer?. Oncotarget, 8, 37200-7.

Forget P, Machiels JP, Coulie PG, et al (2013). Neutrophil:lymphocyte ratio and intraoperative use of ketorolac or diclofenac are prognostic factors in different cohorts of patients undergoing breast, lung, and kidney cancer surgery. Ann Surg Oncol, 20, 650-60.

Franco AT, Corken A, Ware J (2015). Platelets at the interface of thrombosis, inflammation, and cancer. Blood, 126, 582-8.

Gadgeel S, Peters S, Mok T, et al (2018). Alectinib versus crizotinib in treatment-naive anaplastic lymphoma kinasepositive $(\mathrm{ALK}+)$ non-small-cell lung cancer: CNS efficacy results from the ALEX study. Ann Oncol, 29, 2214-22.

Gaspar LE, Gay EG, Crawford J, et al (2005). Limited-stage small-cell lung cancer (stages I-III): observations from the National Cancer Data Base. Clin Lung Cancer, 6, 355-60.

Gaspar LE, McNamara EJ, Gay EG, et al (2012). Small-cell lung cancer: prognostic factors and changing treatment over 15 years. Clin Lung Cancer, 13, 115-22.

Hong X, Cui B, Wang M, et al (2015). Systemic immuneinflammation index, based on platelet counts and neutrophillymphocyte ratio, Is useful for predicting prognosis in small cell lung cancer. Tohoku J Exp Med, 236, 297-304.

Hong X, Xu Q, Yang Z, et al (2018). The value of prognostic factors in Chinese patients with small cell lung cancer: A retrospective study of 999 patients. Clin Respir J, 12, 433-47.

Horn L, Mansfield AS, Szczesna A, et al (2018). First-line atezolizumab plus chemotherapy in extensive-stage small-cell lung cancer. $N$ Engl J Med, 379, 2220-9.

Kasmann L, Bolm L, Janssen S, et al (2017). Prognostic factors and treatment of early-stage small-cell lung cancer. Anticancer Res, 37, 1535-7.

Lee S, Oh SY, Kim SH, et al (2013). Prognostic significance of neutrophil lymphocyte ratio and platelet lymphocyte ratio in advanced gastric cancer patients treated with FOLFOX chemotherapy. BMC Cancer, 13, 350.

Liu D, Huang Y, Li L, et al (2017). High neutrophil-tolymphocyte ratios confer poor prognoses in patients with small cell lung cancer. BMC Cancer, 17, 882 .

Monreal M, Fernandez-Llamazares J, Pinol M, et al (1998). Platelet count and survival in patients with colorectal cancer--a preliminary study. Thromb Haemost, 79, 916-8.

Pedersen LM, Milman N (1996). Prognostic significance of thrombocytosis in patients with primary lung cancer. Eur Respir J, 9, 1826-30.

Pedrazzani C, Mantovani G, Fernandes E, et al (2017). Assessment of neutrophil-to-lymphocyte ratio, platelet-to-lymphocyte ratio and platelet count as predictors of long-term outcome
Platelet Parameters in Limited Disease Small Cell Lung Cancer after R0 resection for colorectal cancer. Sci Rep, 7, 1494.

Riedl J, Pabinger I, Ay C (2014). Platelets in cancer and thrombosis. Hamostaseologie, 34, 54-62.

Siegel RL, Miller KD, Jemal A (2018). Cancer statistics, 2018. CA Cancer J Clin, 68, 7-30.

Silvestri GA, Gonzalez AV, Jantz MA, et al (2013). Methods for staging non-small cell lung cancer: Diagnosis and management of lung cancer, 3rd ed: American College of Chest Physicians evidence-based clinical practice guidelines. Chest, 143, 211-50.

Smyth SS, McEver RP, Weyrich AS, et al (2009). Platelet functions beyond hemostasis. J Thromb Haemost, 7, 1759-66.

Song X, Zhu H, Pei Q, et al (2017). Significance of inflammationbased indices in the prognosis of patients with non-metastatic colorectal cancer. Oncotarget, 8, 45178-89.

Suzuki R, Wei X, Allen PK, et al (2019). Prognostic significance of total lymphocyte count, neutrophil-to-lymphocyte ratio, and platelet-to-lymphocyte ratio in limited-stage small-cell lung cancer. Clin Lung Cancer, 20, 117-23.

Tomita M, Shimizu T, Hara M, et al (2009). Preoperative leukocytosis, anemia and thrombocytosis are associated with poor survival in non-small cell lung cancer. Anticancer Res, 29, 2687-90.

Uemura T, Hida T (2018). Durvalumab showed long and durable effects after chemoradiotherapy in stage III non-small cell lung cancer: results of the PACIFIC study. $J$ Thorac Dis, 10, 1108-12.

Varlotto JM, Recht A, Flickinger JC, et al (2011). Lobectomy leads to optimal survival in early-stage small cell lung cancer: a retrospective analysis. J Thorac Cardiovasc Surg, 142, 538-46.

Xie D, Marks R, Zhang M, et al (2015). Nomograms predict overall survival for patients with small-cell lung cancer incorporating pretreatment peripheral blood markers. J Thorac Oncol, 10, 1213-20.

Zhang H, Liu L, Fu S, et al (2017a). Higher platelet distribution width predicts poor prognosis in laryngeal cancer. Oncotarget, 8, 48138-44.

Zhang Q, Qu Y, Liu H, et al (2019). Initial platelet-to-lymphocyte count as prognostic factor in limited-stage small cell lung cancer. Biomark Med, 13, 249-58.

Zhang X, Cui MM, Fu S, et al (2017b). Platelet distribution width correlates with prognosis of gastric cancer. Oncotarget, 8 , 20213-9.

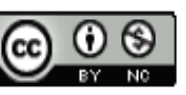

This work is licensed under a Creative Commons AttributionNon Commercial 4.0 International License. 